\title{
Protection System of Communication Line based on Distributed Interfer- ence Fiber Sensing Network
}

\author{
Chunxia $\mathrm{Ma}^{1}$, Yian $\mathrm{Cui}^{2}$ and Guangshun Shi ${ }^{*}$ \\ ${ }^{1}$ Engineering Training Center, Tianjin Polytechnic University, Tianjin, 300387, China \\ ${ }^{2}$ College of Computer and Control Engineering, Nankai University, Tianjin, 300071, China
}

\begin{abstract}
On the issue of security and protection of communication line, this study designed and realized a new distributed fiber vibration sensing and warning system for hazardous events. Based on $\mathrm{M}-\mathrm{Z}$ interference principle, the system realized the real-time detecting and accurate locating of the vibration signals by means of cross-correlation calculation of the fiber signals, restrained effectively the sensing signal noise and extracted accurately the vibration signals of various hazardous events with the methods of wavelet threshold shrinkage and Hilbert transform. In the meantime, the study proposed multi-dimensional features including wavelet decomposition, MFCC coefficients and time-domain envelope to describe the vibration signals and established multi-level classifier models with BP neural network and SVM methods to realize the classification and recognition of various hazardous events. After the applications and experiments of the system on 7 hazardous events, we obtained experimental results of the locating accuracy of $<100$ meters and classifying accuracy rate of $94.35 \%$ on hazardous events, which verified the effectiveness of the study.
\end{abstract}

Keywords: Artificial neural network, Digital signal processing, Fiber sensing network, Security of communication line.

\section{INTRODUCTION}

The protection of communication line has been an important issue for society's communication infrastructure. Currently there have been few researches in security warning and destructive behaviors detect of communication line. Wang [1] has experimented with an acoustic detecting system able to discriminate background noise and hammering signals within a distance of $2.4 \mathrm{~km}$. However, the system has certain limitations that it cannot detect long-distance destructions and unable to locate accurately due to its limited range of sound signal detection. Distributed fiber sensing technology is considered to be an important direction for future development [2]. Secure Pipe System, developed by Future Fiber Technology (FFT) [3, 4], a leading company in the field from Australia, monopolized the technology and has not been introduced to Chinese market. The system proposed by Juarez [5] can only locate signal but unable to analyze the features of the detected signal.

In this context, we conducted a research in the security warning of communication line, and on the basis of former resources, established a low cost and highly reliable protection system of communication line based on distributed fiber sensing network. This system formed a Mach-Zehnder fiber interference system with fiber to sense and transmit vibrating signal, which is dealt as the process object to distinguish whether there are destructive behaviors around the communication lines. If any destruction detected, the system will locate the destruction and distinguish the destruction pattern with neural network and support vector machine (SVM), thus to realize a long-distance security warning. Simulated field experiments of destructive behaviors and the collected and analyzed data showed that the system can distinguish effectively between destructive and non-destructive events. The recognition rate on the 7 destructive behaviors is as high as $94.35 \%$.

\section{ARCHITECTURE OF FIBER SENSING NETWORK}

\subsection{Vibrating Signal Collecting}

As shown in Fig. (1), the protection system of communication line based on distributed fiber sensing network mainly consists of hardware module and software module. Important hardware modules include: light source module, sensing optical path and demodulation module. The main purpose of software system is to detect and process the vibrating signals so that to realize the recognition and warning of the signals. The basic principle of $\mathrm{M}-\mathrm{Z}$ interferometer applied in distributed vibration sensing is to measure the modulation of vibrating signal on light wave phase by interference when light is transmitted in fiber to achieve the detection and location of vibrating signals [6]. As derived from theory [7], when vibrating signal acts on the fiber, the light wave phase in the fiber will be modulated and the vibrating signal can be collected by the detection of the emergent light wave phase.

As shown in Fig. (2) the fiber is laid along the protection object and the light wave sent out by the laser light source was divided into two light beams by fiber coupler, one 


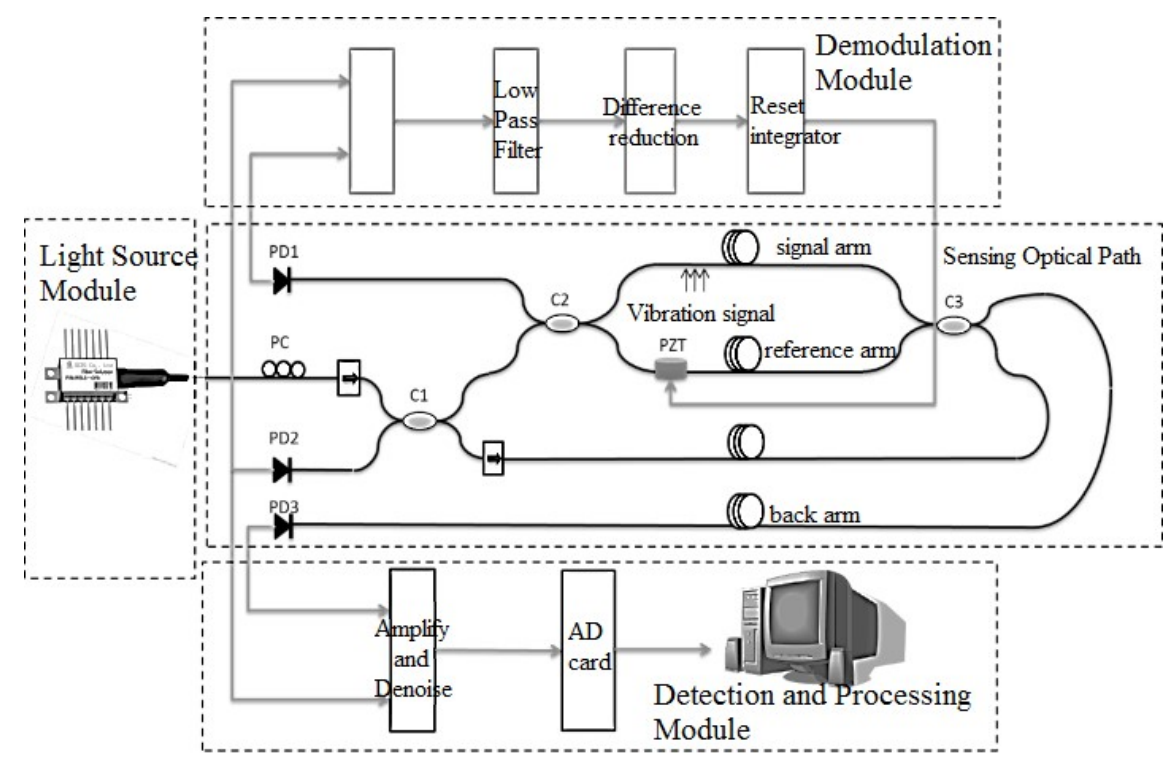

Fig. (1). Schematic diagram of distributed interference fiber line protection system.

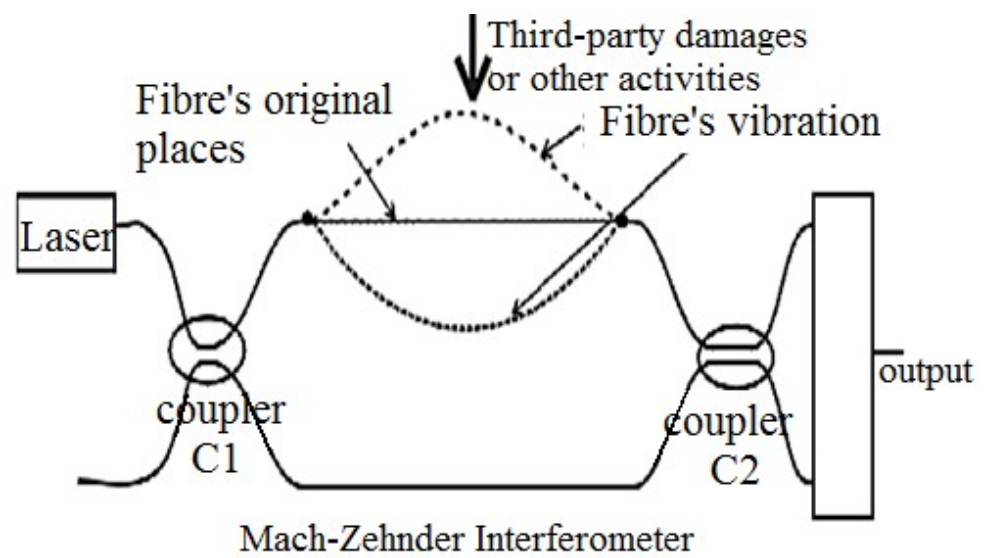

Fig. (2). Schematic view of vibrating signal collecting mechanism.

transmitting in sensing fiber arm and the other transmitting in fiber reference fiber arm.

When a destructive behavior occurs, the sensor fiber will deform with the vibration and the phase will be affected by the deformation and thus modulated. Therefore, the vibrating signal is included in the interference signal phase when the two light beams meet at the coupler and interfere with each other. The vibrating signal can be obtained by measuring the change of light signal intensity, as the phase is changed with the intensity of the emergent light signal from the interferometer. The light signal is converted into photoelectric signal by the photoelectric conversion circuit and sent out from the two output ports of the coupler. The two beams of light are complementary. Based on this theory [6], we can conclude the modulation formula as follow:

$\omega(t)=\frac{4 \pi^{2} n v A^{2}}{\lambda l} \sin (2 \times 2 \pi v t)$

Where, $\omega(t)$ represents the frequency of photoelectric signal, $\lambda$ refers to the wavelength of light source, $l$ refers to the length of fiber, while $v$ and $A$ represent the frequency and vibrating range of outer vibration, respectively. As seen in the formula 1 , the value of photoelectric signal frequency not only has connection with the wavelength of light source, the length of fiber as well as the frequency and vibrating range of outer vibration, but also is modulated by the outer vibrating frequency. Therefore, whenever destructive behavior happens, it can't be recognized simply by calculating frequencies of photoelectric signals.

\subsection{Location of Signal}

The location of vibrating signal cannot be obtained only by a single $\mathrm{M}-\mathrm{Z}$ interferometer but with a double $\mathrm{M}-\mathrm{Z}$ interferometer structure, which can be seen in Fig. (3). The light sent out from LD laser passes $3 \mathrm{~dB}$ coupler and is divided into two beams of light as clockwise light and anticlockwise light. The clockwise light reaches the photoelectric detector PD2 through the back arm and C1, after they were interfered in the $\mathrm{M}-\mathrm{Z}$ interferometer, formed by $3 \mathrm{~dB}$ coupler $\mathrm{C} 2$ and $\mathrm{C} 3$. The anticlockwise light reaches the photoelectric detector PD1 via the back arm and M-Z interferometer. As the light wave in the fiber is two-way transmitted, optoisolators 


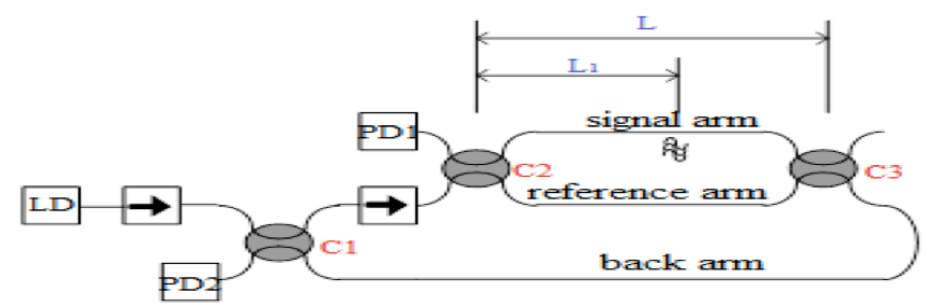

Fig. (3). Location schematic diagram based on double M-Z structure.

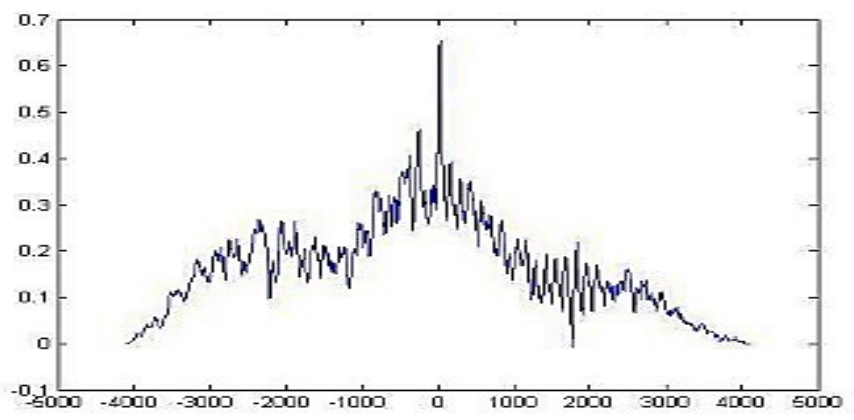

(a)Cross-correlation result of the two effective signals

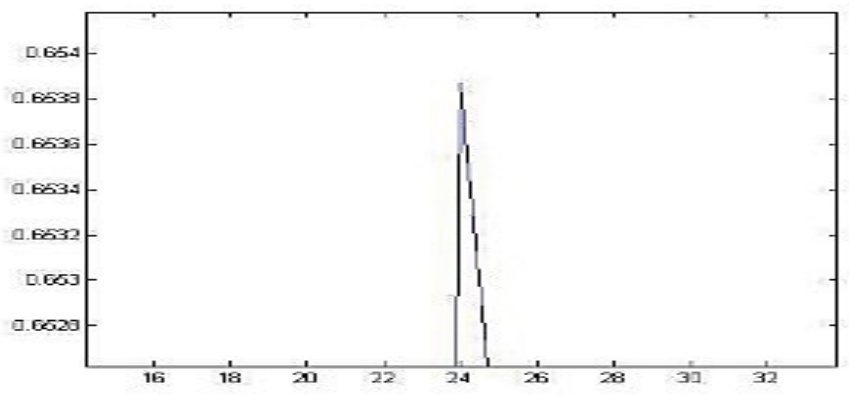

(b) Magnification of the cross-correlation result

Fig. (4). Cross-correlation result and magnification of the two effective signals.

need to be added in $\mathrm{LD}, \mathrm{C} 1$ and $\mathrm{C} 2$, to avoid any anticlockwise light coupling at $\mathrm{C} 1$ and returning to $\mathrm{LD}$. As the clockwise and anticlockwise interferometers employ the same signal arm and reference arm and the phase modulation of vibration on fiber is the same, the light signals received by PD1 and PD2 are exactly the same except with a certain time delay [6].

We assume the length of entire signal arm fiber is L, the vibration happens at the location L1 away from the right coupler, the time that photoelectric detector receives the interference signal will be:

$t_{1}=\frac{L_{1} n}{\mathrm{c}}$

The time that photoelectric detector receives the interference signal for the second time will be:

$t_{2}=\frac{\left(L-L_{1}+L\right) n}{c}=\frac{\left(2 L-L_{1}\right) n}{c}$

Where, $n$ represents the refractive index of fiber medium, $c$ refers to the light speed in vacuum. According to (1) and (2), the time difference of the two photoelectric signals will be:
$\Delta t=t_{2}-t_{1}=\frac{2\left(L-L_{1}\right) n}{c}$

Thus, the location of vibration can be calculated through the time difference of the two signals:

$L_{1}=L-\frac{\Delta t c}{2 n}$

Due to the continuum light source of the system, the absolute value of $t_{1}$ and $t_{2}$ cannot be measured. We can conduct a cross-correlation calculation of the two photoelectric signals. Then the cross-correlation function will produce a maximum value at $\Delta t$ and the location of vibration signal can be realized according to the light speed in the fiber and $\Delta t$.

The signal that photoelectric converting circuit sends out is a $100 \mathrm{kHz}$ sampled signal. The high sampled frequency is to provide enough data to the location module of the vibration source to assure the locating accuracy within 100 meters. The cross-correlation result of the two effective data domain signal (10 k interception points) is shown in Fig. (4) (a). Obviously the peak value of cross-correlation result is sharp, which is helpful in determining the location of maxi- 

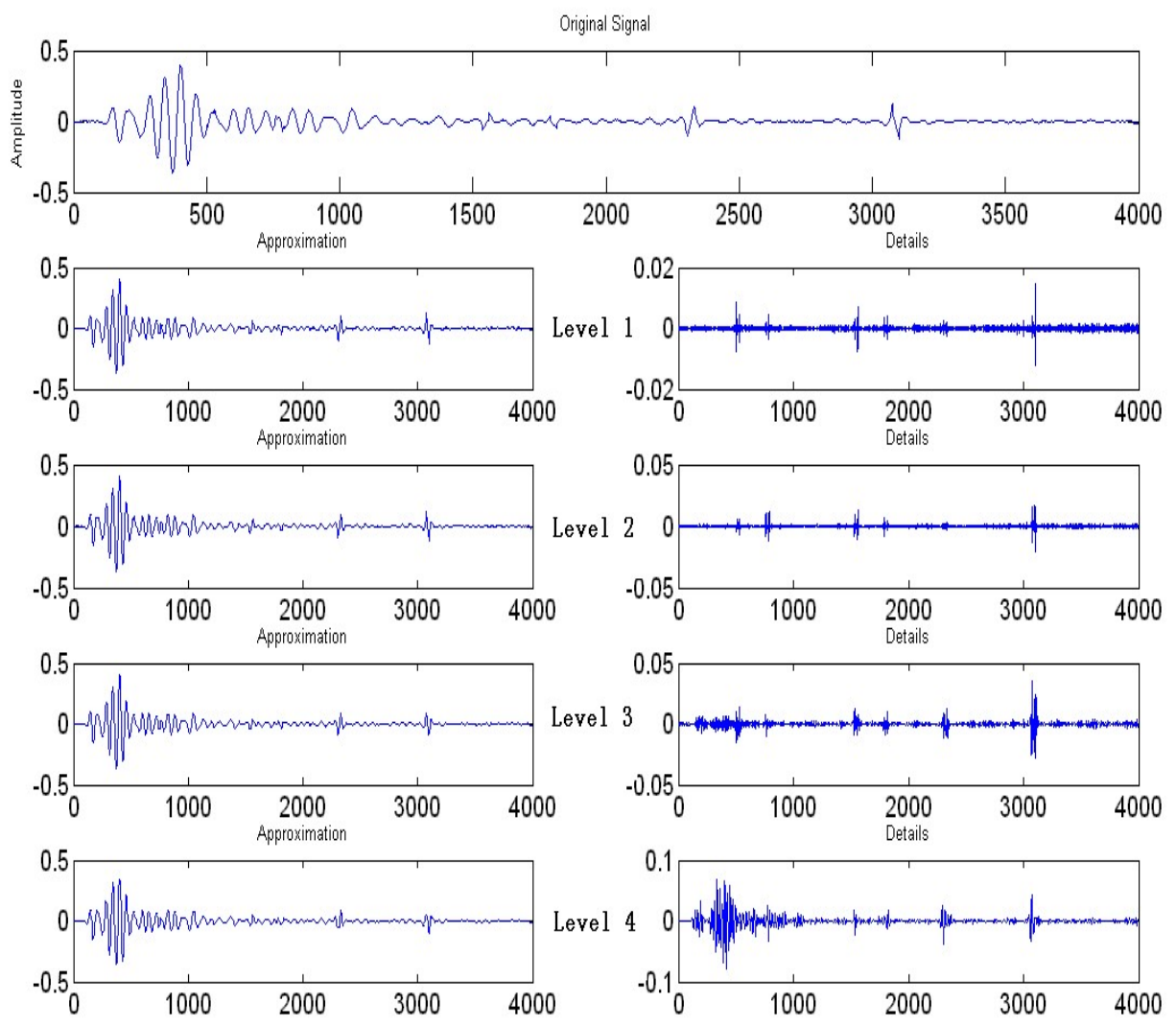

Fig. (5). Discrete Wavelet Analysis of Tamping Signal.

mum value. The relevant time delay of the maximum value location of cross-correlation result is an estimation of the time delay points of the two signals. The locating result of the vibration can be obtained after putting the time delay points into formula (5).

\section{VIBRATION SIGNAL PROCESSING}

\subsection{Noise Reduction of Vibration Signal}

Due to the complicated laying environment of communication line, the collected vibration signal contains a lot of noise that swallows the useful information. Since the frequency distribution width of useful signal is much smaller than that of the noise, we adopted frequency domain filtering method to restrain noise. The band-pass filter, whose frequency range of vibration signal is $50-3000 \mathrm{~Hz}$, enabled the acquisition of clear signal. However, the signal collected with a $50-3000 \mathrm{~Hz}$ band-pass filter is still mixed with large amount of high frequency noise and the energy is mainly lower than $500 \mathrm{~Hz}$. Therefore, the frequency domain information can be further compressed. The Wavelet Analysis [7, 8] Method will be employed in our subsequent processing since it can restrain noise, filter wave, compress the signal and reduce its dimension.

Wavelet Threshold Shrinkage Method reduces noise in accordance with the powerful data-remove correlation of wavelet transformation. In the wavelet domain, this correlation concentrates the signal energy in some large wavelet coefficients and distributes the noise energy in the whole wavelet domain to obtain smaller wavelet coefficients. In this way, there will be only one threshold value that is equal to or larger than the wavelet coefficients of noise. Therefore, a threshold value should be established to compare with all the wavelet coefficients among which the ones smaller than this threshold value should be filtered and the other coefficients which are not smaller than this threshold value should be saved as the wavelet coefficients of useful signal. Then, wavelet reconstruction can be applied to obtain noise-free vibration signal. In Fig. (5), the fourth-order Daubechies wavelet is applied to conduct a four-level wavelet decomposition of the tamping signal. The top image in Fig. (5) shows the original signal that contains noise; images in the second line are the first-level wavelet decomposition, between which the left one is the signal obtained through the reconstruction of low frequency coefficients and the right one obtained with high frequency coefficients reconstruction. The following third and fourth lines are the results of the secondlevel and third-level wavelet decomposition or reconstruction, respectively. In general, the noise energy from the firstlevel to the third-level is low and its energy level is different from its original signal. In the fourth-level decomposition, the energy of high frequency signal in the vibration location increases. Thus, the low frequency part of the third-level decomposition preserves most of the information of the original signal.

\subsection{Segmentation of Vibration Signal}

Since these two collected signals have complementary phases and slight time delay, their correlation can be used to 

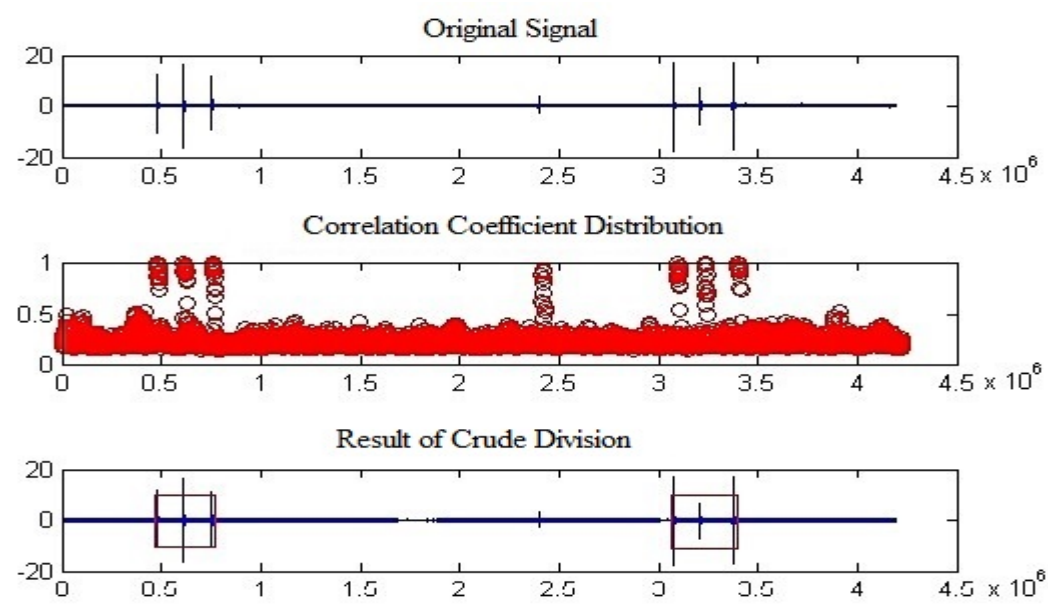

Fig. (6). Crude extraction of correlative coefficients.
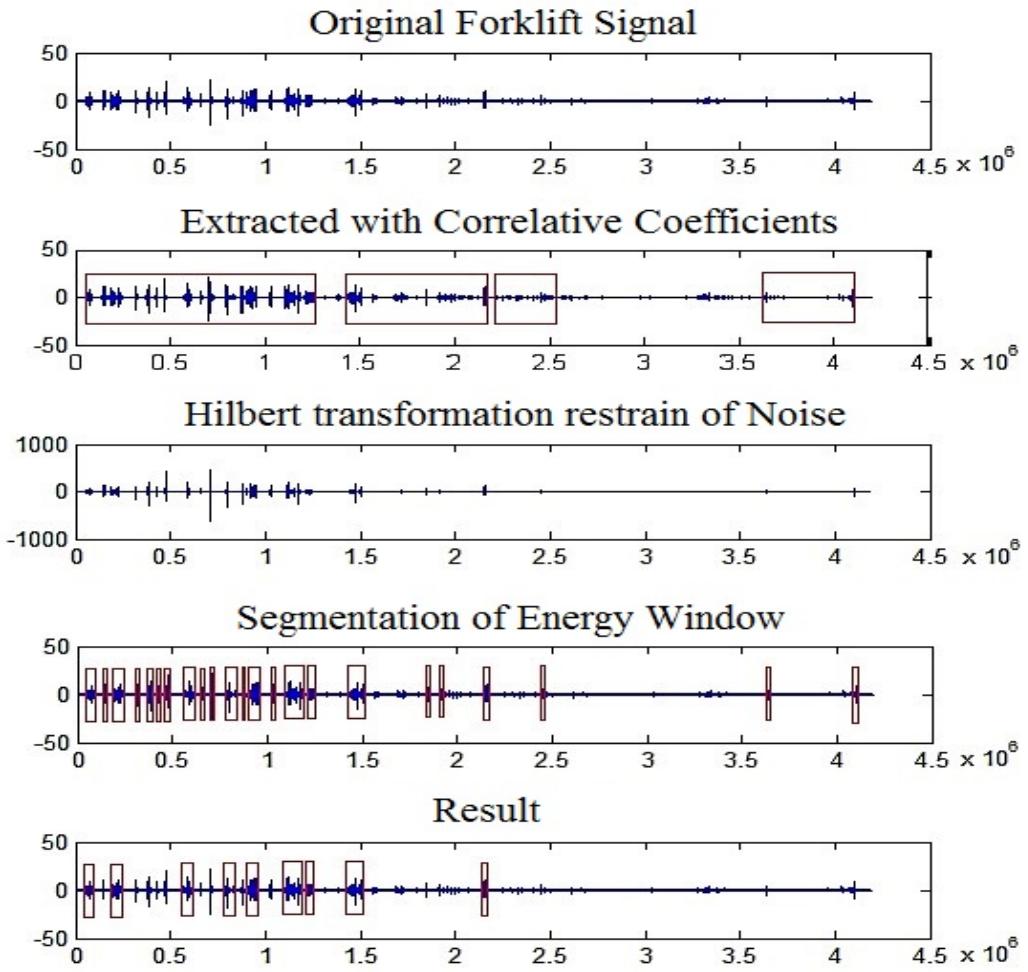

Fig. (7). Segmentation of the Original Forklift signal.

remove the non-vibration signal segments. In autocorrelation analysis, the vibration signal is achieved. Fig. (6) presents the original tamping signal pattern, the distribution diagram of its correlative coefficients and the crude division results (enclosed by rectangular boxes) of correlative coefficients. As shown in the Fig. (6), the crude extraction of correlative coefficients not only perfectly preserved the vibration signal, but also largely reduced the data volume.

Then, the signal envelope is calculated through the Hilbert transformation of the original signal. Multiply the sequence of original signal by the sequence of envelope. The multiplied result shows that the vibrating part of original signal is more distinct and the non-vibrating part (noise) has been effectively restrained.
In certain cases noise is restrained to almost 0 , which counts as dilation and erosion operations of the original signal. Under these circumstances, the difference between vibration energy and noise energy is more distinct. Lastly, the sliding window based energy segmentation algorithm is utilized to further refine the fragment division of signals. In this procedure, window will serve as the unit to calculate timedomain energy of each window. The window energy that surpasses the threshold value are selected as candidate vibrating fragments; if the intervals of candidate vibrating fragments are within certain range, these fragments will be merged into one; if not, they will be preserved as different vibration fragments. Fig. (7) shows the integrated division process of the vibrating and non-vibrating parts of the forklift original signal. 
Table 1. List of vibration signal feature extraction.

\begin{tabular}{|c|c|}
\hline Feature Extraction & Feature \\
\hline \hline J level Wavelet Decomposition $(\mathrm{J}=6)$ & $\mathrm{L}=32$ \\
\hline Frequency Domain Feature-MFCC & $\mathrm{M}=24$ \\
\hline Time Domain Feature Envelope & $\mathrm{K}=30$ \\
\hline
\end{tabular}

\subsection{Feature Defination and Extraction}

Vibration signal is a kind of non-stationary dynamic signal with strong time-variant property. Wavelet transformation is featured with multi-resolution and it is able to characterize signal's local features in both time and frequency domains. Compared with wavelet analysis, wavelet packet is finer in band decomposition; it decomposes the high frequency parts as well. Consequently, wavelet packet analysis is applied in this study. Frequency spectrum is one of the major features of vibration signal. Various vibration signals have been collected and replayed, which indicates that human ears can distinguish between most kinds of vibration events. Therefore, MFCC can be extracted as the vibration signal's features in frequency domain. In addition, vibration signal has distinct features in time domain.

For instance, as discontinuous event and continuous event have different durations, they can be easily separated from each other in accordance with time domain. Besides, the amplitude variation of vibration signal is also a useful factor, whose envelope can be encoded as part of eigenvector.

The signal amplitude itself can be applied as a feature. For tamping signal and digging signal, they share similar distribution in frequency domain but vary widely in amplitude; the force of tamping is 3-5 times stronger than digging. In feature extraction process of vibration signal, the noisefree vibration signal fragments coming from time domain are the extraction objects. To sum up, the feature extraction is achieved from the following three aspects (See Table 1).

\section{RECOGNITION OF VIBRATION SIGNAL}

\subsection{Classifier Settings}

The ultimate goal of security and warning system of communication line is to classify the destructive and safety events. On this basis, we proposed a multi-level classifier model based on neural network. The model takes neural network as the first level classifier to classify the single signal fragment and determine the event category according to the common vote of multi signal fragments belonging to the same event, which can be considered as the second level classification.

Since the input dimension is a little larger in our case, we may not control the space complexity of hidden layer in advance if we select a kernel based classifier such as RBF or SVM. Thus we select the simplest network, namely BP networks. In order to avoid over-learning, we set two nodes in hidden layer. In our implementation, weight and bias values are updated according to basic back-propagation algorithm together with gradient descent momentum and an adaptive learning rate [10]. The updating formula is:

$\Delta \omega=-\eta \nabla_{\omega} \mathrm{E}+\alpha \Delta \omega_{\text {prev }}$

And the rules to update weights are: 1) if MSE over the whole training set increases after weight updating, and the value exceeds a fixed bias $\zeta$, which in our case is set to $5 \%$, then we cancel the updating, and multiply the learning rate with a factor $\rho(0<\rho<1)$, which is set to be 0.8 in our experiment, moreover, momentum parameter $\alpha$ is set to be zero; 2) if MSE decreases after weight updating, then we accept the updating, and multiply the learning rate with a factor $\tau>1$, which is 1.05 in our experiment, moreover, if momentum parameter $\alpha$ has been set to be zero, then reset $\alpha$ to its previous value; 3 ) if MSE increases after weight updating, and the value doesn't exceed $\zeta$, then we accept the updating, and keep the learning rate unchanged. Moreover, we reset $\alpha$ to its previous value if momentum parameter $\alpha$ has been set to be zero.

\subsection{Environment Adaptive Method}

Due to the complicated outdoor environment and impact of the climate and weather, signals of the same event may vary. Our recognition is based on manual neural network and recognized events by current sample learning. But in the real situation, it is difficult to collect the vibrating signal covering all circumstances. As a result, if the classifier trained according to the collected data of a certain location is used in other locations, its classifying performances may be reduced. In addition, the current classifier can only classify finite set of events. There may be other destructive events that may occur in future. Therefore, we proposed the increment learning mechanism. With this module, the whole system will possess the functions of self-adaption and self-learning. As shown in Fig. (8), the increment learning mechanism required manual attendance and data base. Manual attendance is realized by a user interface embedded in the server. And data base contains two sheets, where statistical features of vibrating signal, results of manual amendments categories and amended warning events are recorded.

\section{EXPERIMENTAL RESULTS}

The experimental data was collected from Tianjin Unicom Wangdingdi branch and CNPC Hebei Gu'an experimental field. Tianjin Unicom Wangdingdi branch provided a $6.1 \mathrm{~km}$ long fiber communication line from its office to Tianjin Unicom Jieyuandao branch. The two ends of the fiber were exposed and the middle part was buried underground. We knock the two ends directly to conduct a locat- 


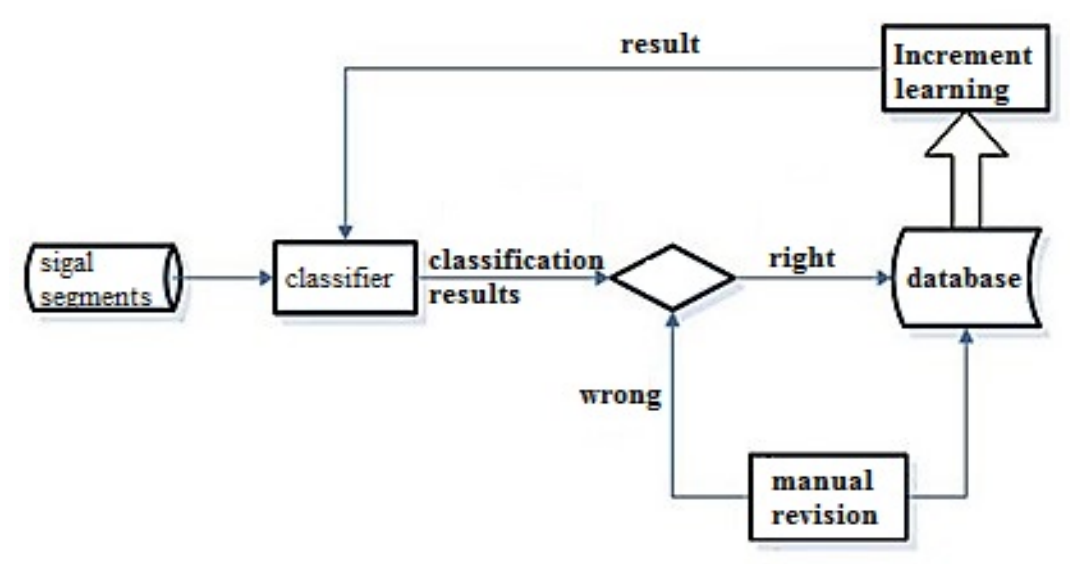

Fig. (8). Schematic diagram of environment adaptive method.

Table 2. The data scale of the 7 unsafe vibrating signals.

\begin{tabular}{|c|c|c|c|c|}
\hline & T & P & F & 68 \\
\hline \hline TRS & 91 & 75 & 34 & 36 \\
\hline TES & 46 & 36 & 102 & 100 \\
\hline TOS & 137 & 111 & 11 \\
\hline
\end{tabular}

Table 2. (continued) The data scale of the 7 unsafe vibrating signals.

\begin{tabular}{|c|c|c|c|}
\hline & W & L & S \\
\hline \hline TRS & 68 & 184 & 36 \\
\hline TES & 34 & 92 & 38 \\
\hline TOS & 102 & 276 & 114 \\
\hline
\end{tabular}

ing data test. The locating accuracy is as much as $100 \mathrm{~m}$. In CNPC Hebei Gu'an experimental field, 56km fiber line was prepared, some of which was placed in silicon tubes and others buried directly underground. The depths include $0.6 \sim 0.8 \mathrm{~m}, 1 \mathrm{~m}, 1.2 \sim 1.3 \mathrm{~m}$ and $1.5 \mathrm{~m} .8$ types of signals were collected, including tamping, piling, farming, electrical drilling, well drilling, large machinery operating, steel pipe hitting and trains. The first 7 types belong to destructive events and trains belong to safety events, which were effectively removed in the signal pretreatment phase and will not affect the subsequent recognitions. The data scale and description can be seen in Table 2 .

As shown in Table 2, we take some fragments from each of the 7 events to form a training set and the rest we keep for a test set. According to the experiment, the recognition rate of SVM on train vibrating signal (T) is $94.86 \%$, which means the safety and unsafety signal recognition rate is $94.86 \%$. The recognition rate of BP neural network on the 7 unsafe vibrating signals (tamping $(\mathrm{T})$, piling $(\mathrm{P})$, farming $(\mathrm{F})$, electrical drilling $(\mathrm{E})$, well drilling $(\mathrm{W})$, large machinery operating (L), steel pipe hitting (S)) is $85.40 \%$. Therefore, the overall recognition rate of the first level on the 8 vibrat- ing signals is $90.38 \%$. The second level is a second amendment of the recognition result of the first level, taking advantage of the recognition result, warning levels and user's rule of them. As shown in Table 3, the recognition rate was increased from $90.38 \%$ to $94.35 \%$ after the second level recognition. In Table 2 , the number of training samples is defined as TRS, the number of testing samples is defined as TES, and the total number of samples is defined as TOS. In Table 3, the number of single events is defined as NSE, number of events in right category is defined as NER and the recognition rate of single category is defined as RSC.

\section{SUMMARY}

On the basis of previous work, this study established a protection system of communication line based on distributed interference fiber sensing network. The experimental result showed that the locating accuracy of the system reaches to $100 \mathrm{~m}$ and the recognition rate of the system on the $7 \mathrm{de}-$ structive behaviors reaches to $94.35 \%$. The system in this study conducted the signal locating with the crosscorrelation method, pretreated the signals with the digital 
Table 3. Experimental results of 2 levels categories of the 8 vibrating events.

\begin{tabular}{|c|c|c|c|c|}
\hline & T & P & F & 113 \\
\hline \hline NSE & 42 & 36 & 33 & 112 \\
\hline NER & 41 & 33 & $93.94 \%$ & $99.12 \%$ \\
\hline RSC & $97.62 \%$ & $91.67 \%$ & S & 67 \\
\hline NSE & W & L & 56 & 62 \\
\hline NER & 88 & 71 & 49 & $87.50 \%$ \\
\hline RSC & $96.59 \%$ & $91.02 \%$ & $92.54 \%$ \\
\hline
\end{tabular}

signal processing method and applied the wavelet analysis in the recognition phase, to make the entire system satisfy the real-time requirement.

At present, although the system has reached a high recognition rate, its warning on serious events needs to be further improved to achieve $100 \%$ accuracy in practical applications.

\section{CONFLICT OF INTEREST}

The authors declared that they have no conflict of interest to this work.

\section{ACKNOWLEDGEMENTS}

Declared none.

\section{REFERENCES}

[1] Q. Wang, C.H. Zhang, Y.F. Geng, and M.Y. Liao, "Damage detection of oil and gas pipeline." Chinese Journal of Scientific Instrument, vol. 22, no. 3, pp. 374-377, 2001.
[2] GTI (Gas Technology Institute),” Pipeline \& Gas Journal, vol. 4, pp. 288-290, 2001

[3] E. Tapanes, "Fibre optic sensing solutions for real-time pipeline integrity monitoring", Australian Pipeline Industry Association National Convention, vol. 3, pp. 27-30, 2001.

[4] E.E. Tapanes, J.R. Goode, and J. Katsifolis, "Apparatus and method for monitoring a structure using a counter-propagating signal method for locating events," Patent 6, vol. 8, no. 17, pp. 717-778, 2004.

[5] J.C. Juarez, and H.F. Taylor, "Distributed fiber optic intrusion sensor system," Journal of Light-wave Technology, vol. 23, no. 6 , pp. 2081-2087, 2005.

[6] Y. Zhou, "Study on the distributed optical fiber sensing technology for pipeline safety detection and location, Tianjin: Tianjin university, pp. 7-38, 2006

[7] G.G. Yen and K.C. Lin, "Wavelet packet feature extraction for vibration monitoring," Industrial Electronics, IEEE Transactions, vol. 47, no. 3, pp. 650-667, 2000.

[8] D.B. Percival, and A.T. Walden "Wavelet methods for time series analysis", Cambridge University Press, pp. 56-150, 2006.

[9] X.L. Xiao," Study on the soil vibration signal analysis and recognition for pipeline", Tianjin: Nankai university, 2008.

[10] M.T. Hagan, H.B. Demuth, and M.H. Beale, "Neural network design", Boston: Pws Publication vol. 12, no. 1, pp. 12-47.

Received: September 22, 2014

Revised: November 04, 2014

Accepted: November 05, 2014

(C) Ma et al.; Licensee Bentham Open.

This is an open access article licensed under the terms of the Creative Commons Attribution Non-Commercial License (http://creativecommons.org/licenses/by-nc/3.0/) which permits unrestricted, non-commercial use, distribution and reproduction in any medium, provided the work is properly cited. 